\title{
Infantile Isolated Absence of Right Pulmonary Artery with an Absent Stump and No Collaterals
}

\author{
Sumant Patil ${ }^{1}$ Sayantan Ghoshbag ${ }^{1}$ Usha Pillay ${ }^{1}$ \\ ${ }^{1}$ Department of Paediatrics, Deenanath Mangeshkar Hospital and \\ Research Centre, Pune, Maharashtra, India \\ J Child Sci 2021;11:e35-e37. \\ Address for correspondence Sumant S. Patil, MBBS, MD, (MRCPCH \\ (UK), DCH(UK), Department of Paediatrics, Deenanath Mangeshkar \\ Hospital and Research Centre, Pune 411004, Maharashtra, India \\ (e-mail: sumantsp22@gmail.com).
}

\author{
Abstract \\ Keywords \\ - unilateral absence of \\ pulmonary artery \\ - UAPA without \\ collaterals \\ - isolated absence of \\ right pulmonary \\ artery
}

Unilateral absence of pulmonary artery (UAPA) is a rare entity. Diagnosis is often incidental based on echocardiography $(\mathrm{ECHO})$ /computed tomography (CT) findings done for a cardiac evaluation or recurrent pulmonary pathology not responding to conventional treatment. Childhood-onset symptoms are often rare, and most of these cases are reported in adulthood or postpuberty. Here, we present a 5-month-old child with an isolated absence of right pulmonary artery without even an arterial stump or collaterals. Treatment options depend on the severity of symptoms and anatomy of the abnormal vessels, and in some cases, patients get better, if not completely well with the available medical and surgical treatments.

\section{Introduction}

Isolated unilateral absence of pulmonary artery (UAPA) is a rare entity and usually undiagnosed at birth as most babies remain asymptomatic. It is not until late childhood or adolescence that clinical manifestations occur. The average age of UAPA patients is 14 years. ${ }^{1}$ UAPA is often associated with other cardiac anomalies; however, it may seldom occur as an isolated lesion which mostly concerns the right pulmonary artery. ${ }^{2}$ Associated defects include patent ductus arteriosus, the tetralogy of Fallot, coarctation of aorta, truncus arteriosus, and pulmonary atresia. ${ }^{3}$ Collaterals to the affected side commonly arise from the bronchial artery but may also arise from intercostal, subclavian, and rarely coronary artery. Diagnosis can be incidental in an asymptomatic child. However, infants generally present with pulmonary hypertension and congestive cardiac failure. Therefore, the treatment must be tailored according to the clinical signs and symptoms, the anatomy of the pulmonary artery, and aortic pulmonary collaterals, as well as the presence of associated cardiovascular anomalies and pulmonary hypertension. ${ }^{4,5}$ Here, we report a case of an infant with isolated UAPA with an absent arterial stump and no collaterals.

received

October 11, 2020

accepted after revision

December 3, 2020
DOI https://doi.org/

$10.1055 / \mathrm{s}-0040-1722567$.

ISSN 2474-5871.

\section{Case Presentation}

A 5-month-old male child was admitted to our hospital with progressive dyspnoea associated with fever and cough for 2 days. This child was born at term with a birth weight of $2.2 \mathrm{~kg}$ and had an uneventful perinatal period. However, this child was not able to gain weight since the first month of life, and his current weight was less than the 5th centile on the World Health Organization (WHO) growth chart.

On arrival at the emergency room, the child was markedly tachypnoeic, with the Downes score of 8. Baseline arterial blood gas revealed metabolic acidosis with anemia and hypoxemia. The child was immediately admitted to the pediatric intensive care unit with oxygen support. Chest examination revealed reduced air entry on both sides; normal heart sounds with regular rate, rhythm, and no murmur. The tip of the liver was just palpable (-Fig. 1).

Sepsis screen was negative but serum troponin $\mathrm{T}$ and serum CPK tests were positive, serum pro-BNP (B-type Natriuretic Peptide) levels were more than 9,000. Twodimensional (2D) echocardiography (ECHO) with color Doppler showed evidence of right atrial dilatation, right ventricular (RV) hypertension, and dysfunction. There was right-to-left flow across a stretched patent foramen ovale

\section{(c) 2021. The Author(s).}

This is an open access article published by Thieme under the terms of the Creative Commons Attribution License, permitting unrestricted use, distribution, and reproduction so long as the original work is properly cited. (https://creativecommons.org/licenses/by/4.0/) Georg Thieme Verlag KG, Rüdigerstraße 14, 70469 Stuttgart, Germany 


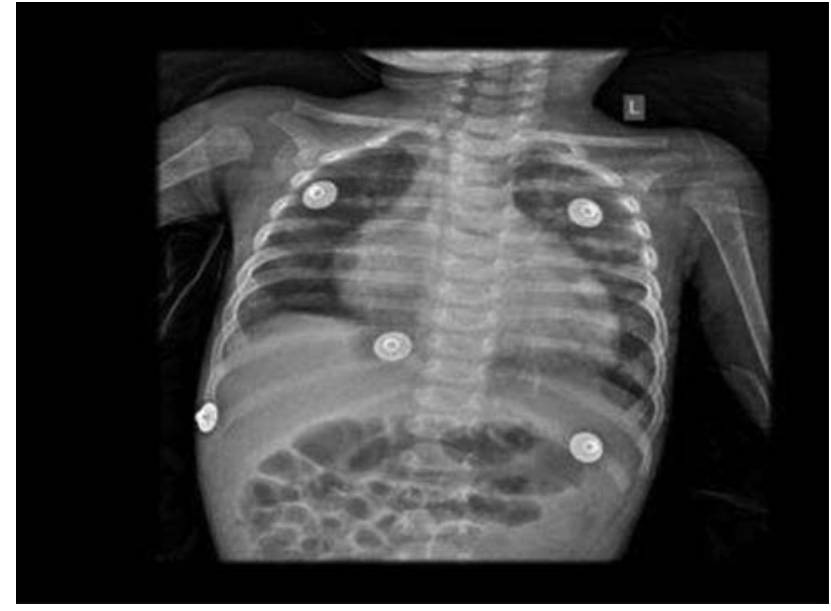

Fig. 1 Chest X-ray: cardiomegaly with mediastinal shift to the right.

(PFO) with no tricuspid regurgitation. The pulmonary veins were of adequate size and connected to the left atrium. Further evaluation revealed a dilated and tense main pulmonary artery (MPA) measuring $1.5 \mathrm{~cm}$ continuing as a dilated left pulmonary artery (LPA) which measured $0.8 \mathrm{~cm}$ in caliber. Right pulmonary artery (RPA) could not be imaged. So, an initial diagnosis of anomalous origin of right pulmonary artery (AORPA)/hemitruncus was suspected. At this stage, absence of a unilateral pulmonary artery was not suspected due to the presence of severe pulmonary hypertension. Computed tomography CT pulmonary angiogram confirmed complete absence of proximal right pulmonary artery with distal artery at the hilum filing through minor collaterals from descending aorta (-Figs. 2-4).

The child was started on antifailure medications while surgical options were being explored. However, after a multidisciplinary meeting, including the cardiac surgical team, we arrived at the conclusion that we cannot offer many remedies surgically. Parents of the child were counselled thoroughly regarding the guarded prognosis with medical treatment. With the ongoing therapy, the child

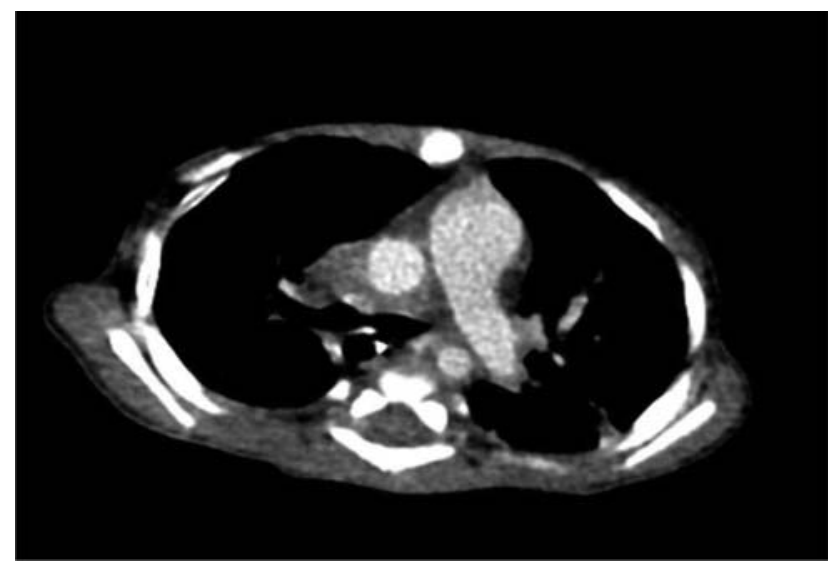

Fig. 2 An axial CT image is showing MPA ${ }^{1}$ and LPA. ${ }^{2}$ RPA is absent. This figure also shows the ascending aorta ${ }^{3}$ and descending aorta. ${ }^{4}$ $\mathrm{CT}$, computed tomography; LPA, left pulmonary artery; MPA, main pulmonary artery; RPA, right pulmonary artery.

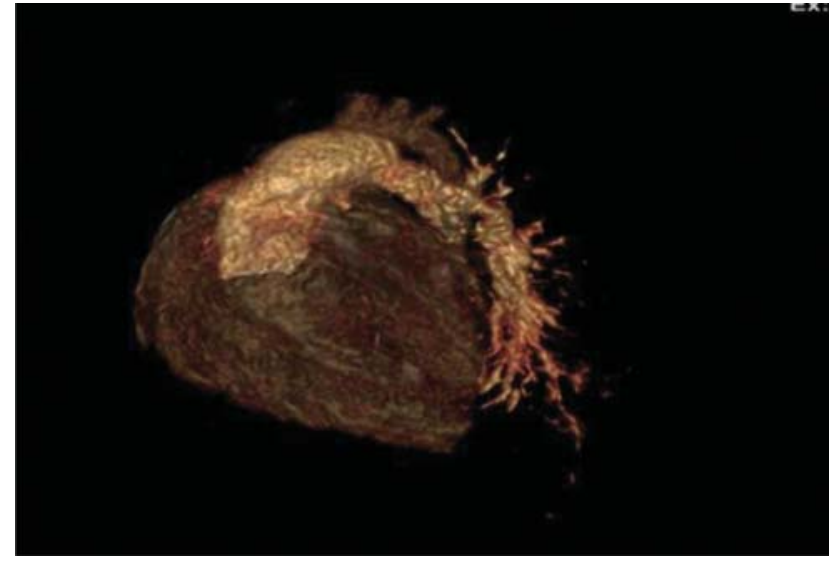

Fig. 3 VRT 3D image of CT pulmonary angiogram showing MPA ${ }^{5}$ and emerging from it, LPA. ${ }^{6}$ RPA cannot be visualized. 3D, three dimensional; CT, computed tomography; LPA, left pulmonary artery; MPA, main pulmonary artery; RPA, right pulmonary artery; VRT, volume rendering technique.

improved symptomatically, though still needed oxygen support.

\section{Discussion}

Isolated UAPA is quite a rare entity with prevalence in the general population ranging from 1 in 200,000 to 1 in 300,000. It often presents with recurrent pulmonary infections and hemoptysis and is known to be associated with other heart defects. ${ }^{4,5}$ The anatomy of UAPA relates to the maldevelopment of the pulmonary artery from the sixth aortic arch during embryogenesis, wherein the proximal part of pulmonary vasculature regresses at the time of birth, and the distal part gets supplied by collaterals from bronchial, intercostal, internal mammary, subdiaphragmatic, subclavian, or even coronary arteries. ${ }^{6}$

Patients with isolated UAPA can have varied presentations ranging from chest pain, pleural effusion, dyspnoea, exercise intolerance, hemoptysis, and pulmonary hypertension.

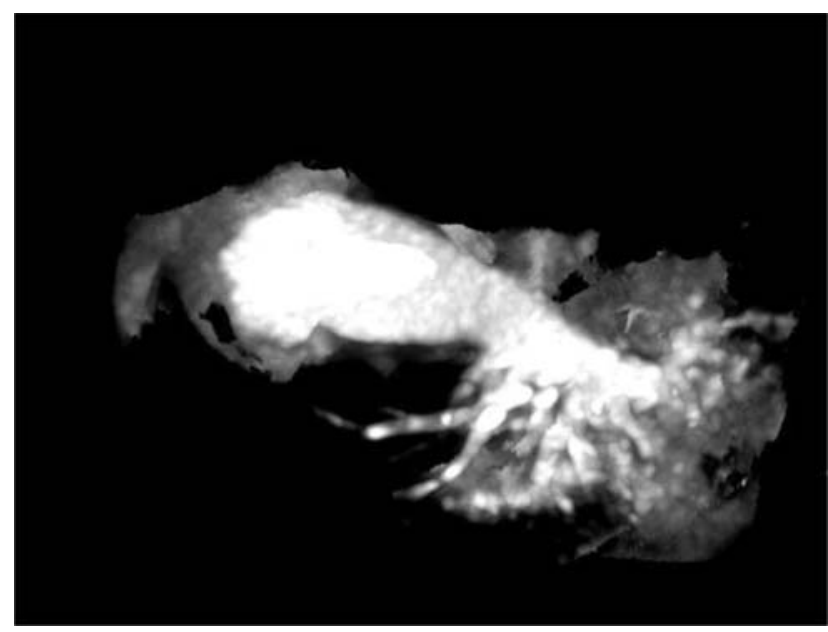

Fig. 4 MIP axial image of CT angiogram revealing $M P A^{8}$ and $L P A^{9}$ while RPA with its stump and collaterals are absent. CT, computed tomography; LPA, left pulmonary artery; MIP, maximum intensity projection; MPA, main pulmonary artery; RPA, right pulmonary artery. 
Therapeutic strategies are based on symptoms of the patient, pulmonary artery anatomy, and associated aortopulmonary collaterals. No treatment is required in patients without evidence of cardiac dysfunction. They only need regular follow-up. ${ }^{7}$

Early detection of an absent pulmonary artery is desirable because it offers the opportunity of surgical repair. The surgical approach has been either creation of an aortopulmonary shunt or connection of the affected pulmonary artery to the main pulmonary artery. However, cases with absent pulmonary artery collaterals are limited and have poor surgical outcomes. ${ }^{2}$ The diagnosis of isolated UAPA is a big challenge for the pediatricians and intensive care teams as the signs are subtle and can be missed. The 2D ECHO is usually normal in patients with isolated UAPA. In contrast, transthoracic color Doppler and 2D ECHO are useful tools to diagnose this malformation and the eventually associated anomalies. Other investigations, such as magnetic resonance imaging (MRI) or CT arteriography, are necessary to confirm the diagnosis and to better describe the pulmonary vasculature. ${ }^{8}$

Limited literature is available on infantile isolated UAPA. Hence, no specific consensus guidelines are available to date regarding the treatment and follow-up of these children. Serial ECHO is recommended to monitor asymptomatic cases for disease progression or development of pulmonary hypertension. ${ }^{9}$ Patients who develop pulmonary hypertension can be treated medically with vasodilator therapy., ${ }^{3,10}$ Alternatively, revascularization of peripheral branches of the affected pulmonary artery to the pulmonary hilum can be attempted, and there are reports of successful revascularization procedures, mostly in the pediatric population. ${ }^{2,11} \mathrm{~A}$ similar case of isolated UAPA was documented in a 4-year-old child who was diagnosed during investigations for repeated lower respiratory tract infections and did not have any definitive surgical option, hence being closely followed-up by pediatricians and cardiologists. ${ }^{12}$

\section{Conclusion}

Isolated absence of the right pulmonary artery is a rare entity. In infancy, it presents mostly with respiratory distress and severe pulmonary hypertension. Diagnosis can be made based on history, physical examination, chest X-ray, ECHO, and CT chest. However, MRI or CT angiography can confirm the ECHO diagnosis and delineate the pulmonary artery anatomy. Treatment is unclear and is based on symptoms and associated conditions and decided by an expectant medical/surgical approach. Our case had an absent right pulmonary artery even with stump being absent and no collaterals, leading to a paucity of surgical options.

Conflict of Interest

None declared.

\section{References}

1 Mohan V, Mohan B, Tandon R, et al. Case report of isolated congenital absence of right pulmonary artery with collaterals from coronary circulation. Indian Heart J 2014;66(02): 220-222

2 Welch K, Hanley F, Johnston T, Cailes C, Shah MJ. Isolated unilateral absence of right proximal pulmonary artery: surgical repair and follow-up. Ann Thorac Surg 2005;79(04):1399-1402

3 Ten Harkel AD, Blom NA, Ottenkamp J. Isolated unilateral absence of a pulmonary artery: a case report and review of the literature. Chest 2002;122(04):1471-1477

4 Bockeria LA, Makhachev OA, Khiriev TKh, Abramyan MA. Congenital isolated unilateral absence of pulmonary artery and variants of collateral blood supply of the ipsilateral lung. Interact Cardiovasc Thorac Surg 2011;12(03):509-510

5 Bouros D, Pare P, Panagou P, Tsintiris K, Siafakas N. The varied manifestation of pulmonary artery agenesis in adulthood. Chest 1995;108(03):670-676

6 Kadir IS, Thekudan J, Dheodar A, Jones MT, Carroll KB. Congenital unilateral pulmonary artery agenesis and aspergilloma. Ann Thorac Surg 2002;74(06):2169-2171

7 Reading DW, Oza U. Unilateral absence of a pulmonary artery: a rare disorder with variable presentation. Proc Bayl Univ Med Cent 2012;25(02):115-118

8 Kruzliak P, Syamasundar RP, Novak M, Pechanova O, Kovacova G. Unilateral absence of pulmonary artery: pathophysiology, symptoms, diagnosis and current treatment. Arch Cardiovasc Dis 2013; 106(8,9):448-454

9 Turner D, Vincent J, Epstein M. Isolated right pulmonary artery discontinuity. Images Paediatr Cardiol 2000;2(03):24-30

10 Shostak E, Sarwar A. A 50-year-old woman with dyspnea, lower extremity edema, and volume loss of the right hemithorax. Chest 2009;136(02):628-632

11 Raymond A, Pedretti E, Privitera G, Cicero C, Biasucci G. Neonatal diagnosis of isolated absence of the right pulmonary artery: a case report and review of the literature. Ital J Pediatr 2018;44(01):27

12 Weldetsadik AY, Asfaw YM, Tekleab AM. Isolated absence of right pulmonary artery in a 4-year old child: a case report. Int Med Case Rep J 2018;11:297-301 